\title{
Bullous Pemphigoid in Children: Clinical Presentation, Pathology and Management
}

\author{
Faris Oumeish ${ }^{1}$, Hamdi Hamad ${ }^{1}$, Hussain Tukmatchy ${ }^{2}$, Niall Scully ${ }^{3}$ and Mohammed Al Abadie ${ }^{*}$ \\ ${ }^{1}$ Department of Dermatology, Royal Wolverhampton NHS Trust, United Kingdom \\ ${ }^{2}$ Hanley Primary Care Centre, London, United Kingdom \\ ${ }^{3}$ Department of Pathology, Royal Wolverhampton NHS Trust, United Kingdom
}

Received: March 19, 2021; Accepted: March 25, 2021; Published: March 29, 2021

*Corresponding author: Mohammed Al Abadie, Royal Wolverhampton NHS Trust, Wolverhampton, United Kingdom; E-mail: mohammed.abadie@nhs.net

\begin{abstract}
Bullous pemphigoid rarely presents in childhood age group and when it presents it has two age peaks infantile and childhood forms. Key presenting features of childhood BP include subepidermal blistering, eosinophilia and severe itching.The histological features of bullous pemphigoid are the same as those described in adulthood and vary with the age of the lesion.Childhood BP tends to have a generally good prognosis with appropriate therapy
\end{abstract}

Keywords: Bullous Pemphigoid; Autoimmune Disorders; Blistering Diseases

\section{Abstract}

Bullous Pemphigoid (BP) is an acquired autoimmune subepidermal blistering disease affecting the elderly and rarely presenting in childhood. It is characterized by the presence of circulating autoantibodies targeting distinctive antigenic components of skin and mucosal basement membrane such as the 180-kDa BP antigen (type XV11 collagen)[1]. Key presenting features of childhood BP include subepidermal blistering, eosinophilia and severe itching. The incidence of BP generally increases with age, unlike other autoimmune diseases, with a variable age of onset in children from 2 months to 15 years [2]. There appears to be no predilection for gender or race and no association with underlying neoplasms. In this literature review, we will explore case reports over the last 20 years to highlight the key clinical and diagnostic characteristics of childhood BP and its management, as well as share our experience in treating a 4 year old boy diagnosed with childhood BP.

\section{Clinical Characteristics}

Childhood BP appears to broadly have two age peaks: infantile and childhood forms. The infantile form occurs in the first year of life and presents predominantly with palmoplantar lesions with or without generalized blistering and rarely with any genital lesions [3].

The childhood form peaks around the age of 8 and has a less uniform presentation of lesions with a higher involvement of the external genitalia in up to $44 \%$ of cases [4]. The two key distinguishing features of childhood BP from adulthood BP include acral involvement and mucous membrane involvement [5].

\section{Infantile form}

Petronius and Bergman reported a 4-month old girl who presented with an erythematous itchy eruption on her extremities spreading rapidly to the trunk, face, and scalp. This presentation was followed by large, tense blisters in same distribution [6]. Similarly, Waisbourd-Zinman et al. reported a case of a 3-month old female infant who presented with an erythematous urticarial papular rash that evolved to develop multiple vesicles on her soles and palms [3]. Martinez-De Pablo et al., reported a 5-month old boy with a similar vesicular eruption in acral distribution associated with oral lesions. They also report three other similar cases without any mucosal involvement [7]. According to Waisbourd-Zinman et al., acral involvement is significantly more likely in infantile than childhood age groups [3].

\section{Childhood form}

In the childhood form tense bullae coexist with urticarial plaques; however there appears to be more involvement of the face and external genitalia. These findings were reported by Belzile, Erbagci and Chou for children with an age range of 20 months to 12 years old $[4,8,9]$. Erbagci described the involvement of the vulva, perianal region in addition to the groins and flexures in their reported case [8]. Waisbour-Zinman suggested a higher likelihood of genital involvement of the disease amongst girls than boys in the childhood subgroup of BP [3].

Three case reports suggested that immunizations may be direct triggers for the bullous eruption, namely Diphteria, Tetanus and Pertussis (DTP), recombinant Hepatitis B vaccine and the mumps, Measles and Rubella (MMR) vaccine $[8,10]$. 


\section{Diagnostic features}

Nemeth et al., outlined diagnostic criteria for childhood BP: 1) Age below 18 years; 2) Presence of vesicles/bullae on clinical examination; 3) Evidence of subepidermal blisters with eosinophilia on histology; 4) Linear deposition of IgG or C3 at the basement membrane zone on Direct Immunofluorescence (DIF) or the presence of anti-basement membrane autoantibodies on indirect immunofluorescence [11].

The histological features of bullous pemphigoid are the same as those described in adulthood and vary with the age of the lesion. Early changes include dermal oedema, perivascular lymphohistiocytic inflammation with eosinophils and eosinophilicspongiosis. Typical established blisters show subepidermal blistering with abundant inflammatory cells including eosinophils within the blister. Rarely the blisters can be cell poor (non-inflammatory) [12]. The histological features can overlap with other diseases within the clinical differential diagnosis such as linear IgA bullous dermatosis and epidermolysis bullosa acquisita. Linear deposition of IgG on DIF is crucial in differentiating from linear IgA bullous dermatosis, hence the importance of histology and DIF in the diagnosis of BP. Voltan et al., also suggests the use of immunoblot assays to determine autoantibodies against the antigens BP180 and BP230, to help differentiate BP from other immunobullous conditions such as linear IgA bullous disease and bullous systemic lupus erythematosus[5]. ELISA testing has also been widely used to detect antibodies for the main antigens in BP: BP180 and 230 kDa BP230 to further confirm the diagnosis of BP [1]. However, comparing ELISA results is challenging due to the variability of commercial and non-commercial ELISA systems deployed in studies in literature. Routine laboratory results are often mixed according to numerous studies with some reporting leukocytosis, eosinophilia, or neutrophilia[3,7,14].

\section{Management}

In our experience we treated a 4 year old boy who was seen as a ward referral after presenting with a severe itchy blistering rash that started 4 days before referral. The blisters started on his hands and feet and extended to the rest of his limbs and trunk[Figure1].

No mucosal involvement was noticed. A clinical diagnosis of childhood bullous pemphigoid was made. He was started on Prednisolone 30mg daily, Erythromycin 500mgBD, Dermovate ointment BD. The blistering rash improved but he continued to develop new blisters and was referred to a paediatric tertiary centre where a biopsy was taken.

Two $4 \mathrm{~mm}$ punch biopsies were taken from the lower abdomen for light microscopic examination and immunofluorescence studies[Figure 2, 3]. The biopsy showed a sharply demarcated subepidermal blister with no significant inflammatory cell population within the blister. The superficial dermis showed minimal perivascular chronic inflammation with only rare eosinophils. Immunofluorescence showed strong linear staining along the dermo-epidermal junction with C3 and weak linear staining with Ig. IgA and IgM were negative. Although the morphological findings were not typical, following discussion at the multidisciplinary meeting the subepidermal blistering with strong linear C3 and weak linear IgG staining was felt best to represent a cell poor bullous pemphigoid.

Following the biopsy result azathioprine $40 \mathrm{mg}$ daily and dapsone $30 \mathrm{mg}$ daily were added to his treatment with good response. Clinical follow-up has demonstrated no new blisters, with resolution of all the original blisters leaving extensive postinflammatory hyperpigmentation [Figure $4 \mathrm{~A}$ and $\mathrm{B}$ ].

There remains no clear guideline for the treatment of childhood BP. Childhood BP may resolve spontaneously due to its benign clinical course with infrequent relapses [13]. Childhood BP tends to have a generally good prognosis with appropriate therapy. Mild or localized forms of the disease have been historically managed by topical steroids; however, more severe forms require a more systemic approach [5]. Systemic oral corticosteroids remain the mainstay of treatment, with numerous studies suggesting good or complete resolution of symptoms [1,5]. Examples of treatment regimens include prednisolone starting at $1 \mathrm{mg} / \mathrm{kg} / \mathrm{d}$ often increasing according to response, methylprednisolone and betamethasone [1,5]. Furthermore, systemic steroid therapy helps in preventing the formation of newer lesions maintaining a degree of sustained remission [14].

In refractory cases, sulphonamides, such as dapsone $1 \mathrm{mg} / \mathrm{kg} / \mathrm{d}$, and other antibiotics such as erythromycin have been used as adjuncts to systemic steroids with good responses. Motegi et al., reported complete resolution of symptoms of their single case with Diaminodiphenyl Sulfone(DDS) at $75 \mathrm{mg} /$ day and maintained remission for a year post-therapy [15]. Xiao et al., reported a case of 14-week-old baby who was given IV immunoglobulin $400 \mathrm{mg} /$ $\mathrm{kg} /$ day course that helped resolve the symptoms within one week, however several relapses did occur throughout the year after treatment [10]. Erbagci opted to add azathioprine to prednisolone and antibiotics to finally achieve remission in their single case [8]. Other studies reported the use of immunomodulators such as mycophenolate, cyclosporin,methotrexate with variable success [9]. Schulze et al., reported a case of a 5 month old boy diagnosed with BP which responded poorly to topical and oral steroid therapy, dapsone, immunomodulators (cyclosprin A and mycophenolatemofetil), and IVIG. Rituximab, monoclonal antibody against the cD20 component of B-Cell surface, was given as a single infusion with almost complete resolution within 2 days. Remission was maintained after a second smaller infusion dose of Rituximab. This study raises the possibility and safety of the use of biologicals in severe refractory cases of childhood BP [16]. 

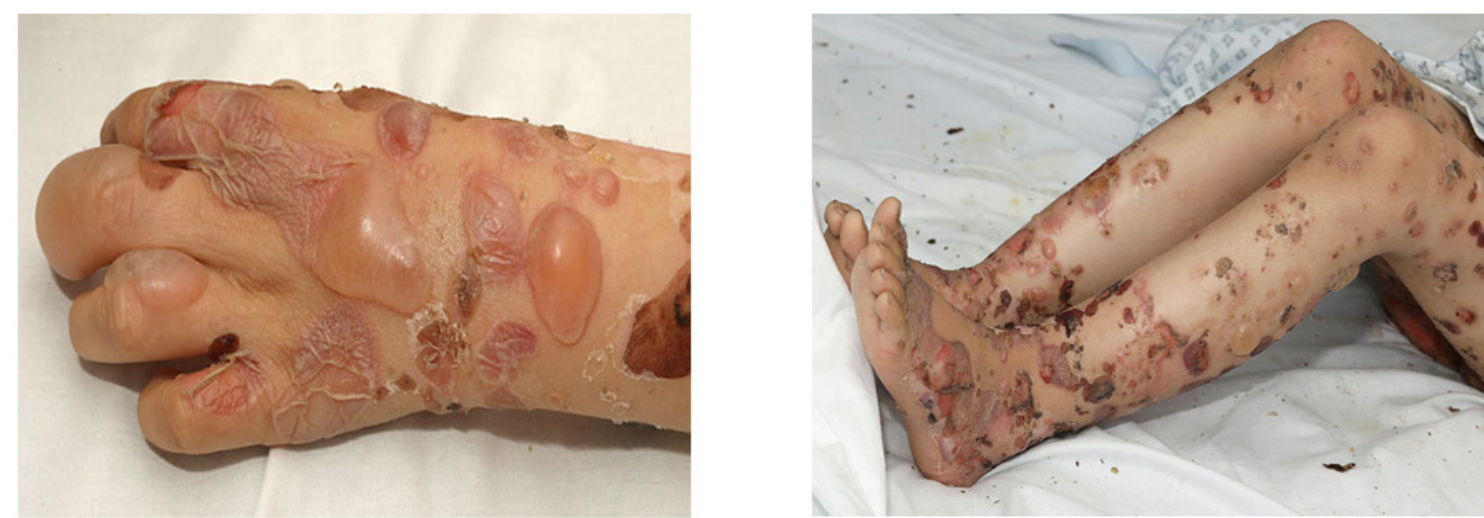

Figure 1(A, B): (A) Tense blisters on the hand and fingers

(B) Tense and flaccid blisters on legs and feet

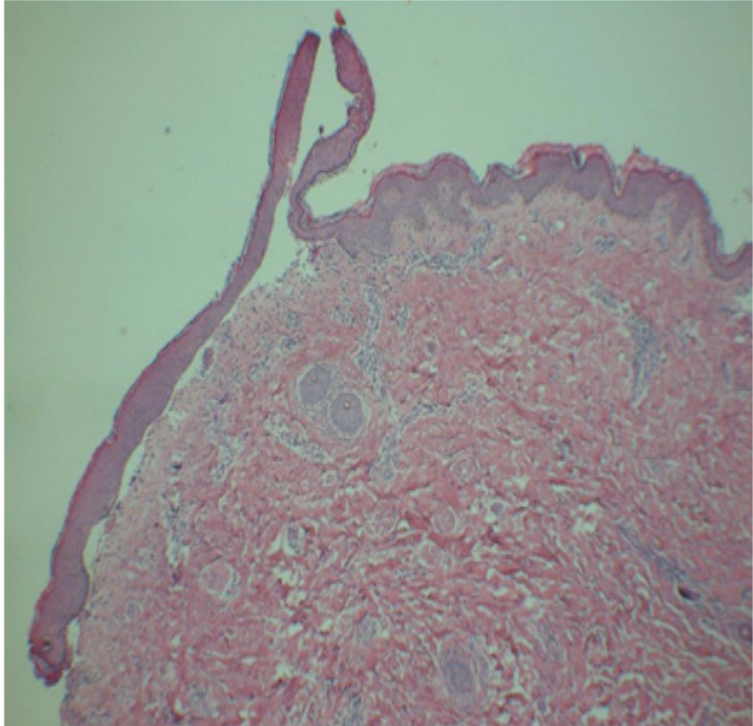

Figure 2: (A) Low power image of sharply demarcated blister with no significant inflammatory component

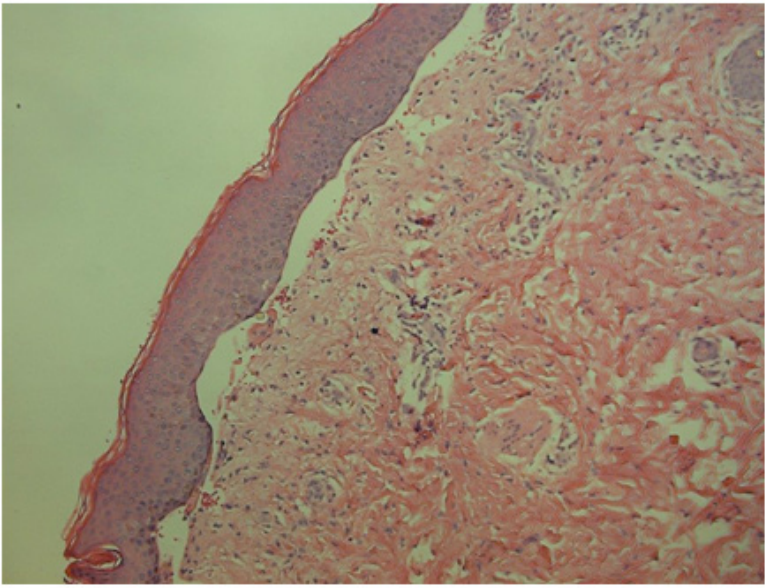

Figure 3: High power image of subepidermal blister with no significant inflammatory component and only mild perivascular chronic inflammation

Citation: Faris Oumeish, Hamdi Hamad, Hussain Tukmatchy, Niall Scully and Mohammed Al Abadie (2021). Bullous Pemphigoid in Children: Clinical Presentation, Pathology and Management. Clin Res Dermatol Open Access 8(1): 1-4. DOI: 10.15226/2378- 

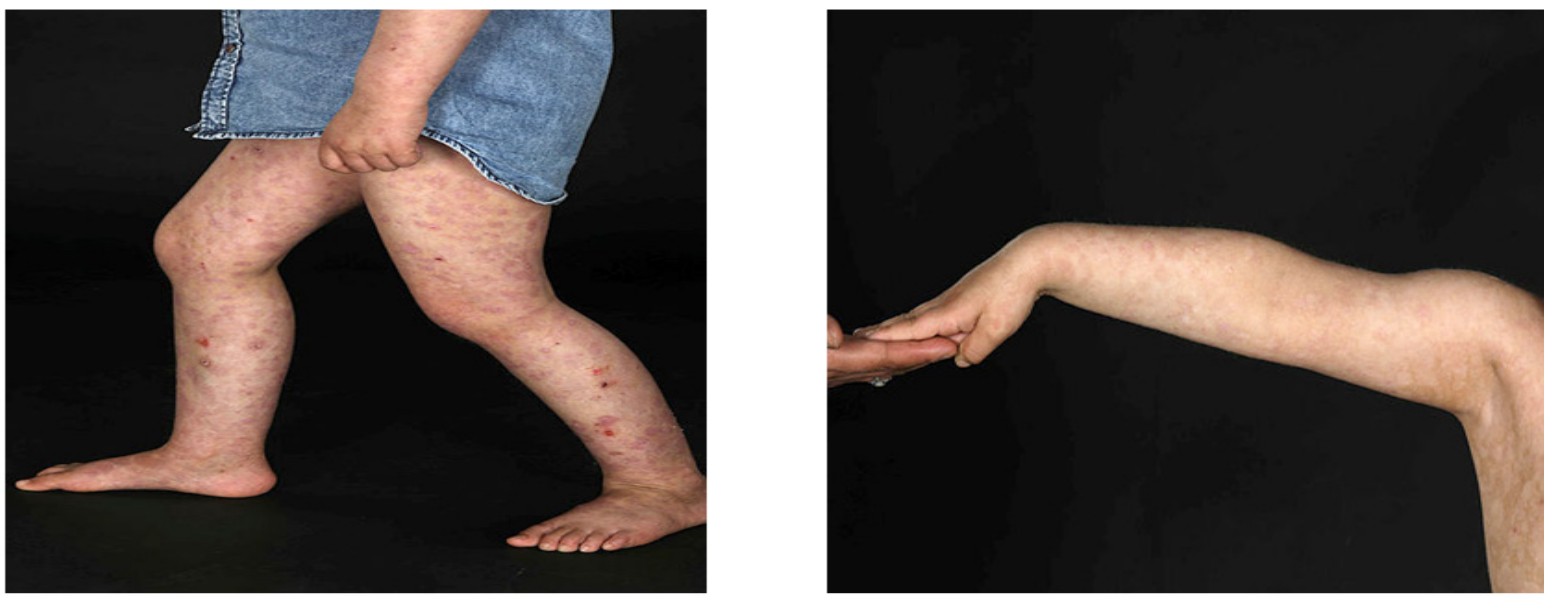

Figure 4 (A)

\section{Conclusion}

Bullous pemphigoid is a rare autoimmune blistering disease in childhood. The histological features are similar to those seen in adulthood form. Childhood BP has a good prognosis with systemic oral corticosteroids remains the mainstay of treatment which in most of the cases leads to complete resolution.

\section{Refernces}

1. Briel A, Moellmann C, Mattulat B, Schauer F, Kiritsi D, Schmidt E, et al. Bullous pemphigoid in infants: characteristics, diagnosis and treatment. Orphanet J Rare Dis. 2014;9(1):185.

2. Weston WL, Morelli JG, Huff JC. Misdiagnosis, Treatments, and Outcomes in the Immunobullous Diseases in Children. Pediatr Dermatol. 1997;14(4):264-272.

3. Zinman O, Amitai D, Cohen, Arnon D, Feinmesser M, Mimouni D, et al. Bullous pemphigoid in infancy: Clinical and epidemiologic characteristics. J Am Acad Dermatol. 2008;58(1):41-48.

4. Belzile E, Funaro D, Powell J. Localized vulvar bullous pemphigoid of childhood: A rare cause of persistent vulvar erosions in children. Pediatr Dermatol. 2019;36(3):349-351.

5. Voltan E, Maeda JY, Silva MAM, Maruta CW, Santi CG, Zimbres S, et al. Childhood Bullous Pemphigoid: Report of Three Cases. J Dermatol. 2005;32(5):387-392.

6. Petronius D, Bergman R. Bullous Pemphigoid in Two Young Infants. Pediatr Dermatol. 2002;19(2):119-121.

7. De Pablo MI, Enseñat MA, Vicente A, Gilaberte M, Mascaró JM. Childhood bullous pemphigoid: clinical and immunological findings in a series of 4 cases. Arch Dermatol. 2007;143(2):215-220.

8. Erbagci Z. Childhood Bullous Pemphigoid Following Hepatitis B Immunization. J Dermatol. 2002;29(12):781-785.

9. Wu KG, Chou CS, Hsu CL, Lee ML, Chen CJ, et al. Childhood Bullous Pemphigoid: A Case Report and Literature Review. J Clin Exp Dermatol Res. 2013;S6:010. doi:10.4172/2155-9554.S6-010

10. Xiao T, Li B, Wang Y, He C, Chen H. Childhood bullous pemphigoid treated by i.v. immunoglobulin. J Dermatol. 2007;34(9):650-653.
11. Nemeth AJ, Klein AD, Gould EW, Schachner LA. Childhood bullous pemphigoid. Clinical and immunologic features, treatment, and prognosis. Arch Dermatol. 1991;127(3):378-386

12. Calonje Eduardo, Brenn Thomas, Lazar Alexander, Phillip H. McKee's pathology of the skin: with clinical correlations. 4th ed. ed. Elsevier/ Saunders. 2012:1803.

13. Reis-Filho E, Silva TdA, Aguirre, Lima, Reis CMS. Bullous pemphigoid in a 3-month-old infant: case report and literature review of this dermatosis in childhood. An Bras Dermatol. 2013;88(6):961-965.

14. Sáenz AM, González F, Cirocco A, Tacaronte IM, Fajardo JE, Calebotta A. Childhood bullous pemphigoid: a case report and 10-year follow up. Int J Dermatol. 2007;46(5):508-510.

15. Motegi S, Abe M, Tamura A, Ishii N, Hashimoto T, Ishikawa O. Childhood Bullous Pemphigoid Successfully Treated with Diaminodiphenyl Sulfone. J Dermatol. 2005;32(10):809-812.

16. Schulze J, Bader P, Henke U, Rose MA, Zielen S. Severe Bullous Pemphigoid in an Infant - Successful Treatment with Rituximab. Pediatr Dermatol. 2008;25(4):462-465. 